\title{
Geomagnetic paleosecular variation for the past 5 Ma in the Society Islands, French Polynesia
}

\author{
Y. Yamamoto ${ }^{1 *}$, K. Shimura ${ }^{1}$, H. Tsunakawa ${ }^{1}$, T. Kogiso ${ }^{2}$, K. Uto ${ }^{3}$, H. G. Barsczus ${ }^{4}$, H. Oda ${ }^{3}$, T. Yamazaki ${ }^{3}$, and E. Kikawa ${ }^{5}$ \\ ${ }^{1}$ Department of Earth and Planetary Sciences, Tokyo Institute of Technology, Meguro-ku, Tokyo 152-8551, Japan \\ ${ }^{2}$ Institute for Frontier Research on Earth Evolution, 2-15 Natsushima-cho, Yokosuka, Kanagawa 237-0061, Japan \\ ${ }^{3}$ Geological Survey of Japan, AIST, Tsukuba Central 7, Tsukuba, Ibaraki 305-8567, Japan \\ ${ }^{4}$ ISTEEM, University de Montpellier II, Montpellier, France \\ ${ }^{5}$ Japan Marine Science and Technology Center, 2-15 Natsushima-Cho, Yokosuka, Kanagawa 237-0061, Japan
}

(Received December 28, 2001; Revised June 20, 2002; Accepted June 21, 2002)

\begin{abstract}
We report a paleomagnetic secular variation in the Society Islands, French Polynesia for the past 5 Ma. Paleomagnetic measurements were performed on volcanic rocks applying thermal and alternating field demagnetizations and principal-component analysis. As a result, 82 normal, 48 reversed, and 10 intermediate directions were observed after the correction of the site localities for the absolute motion of the Pacific plate. A mean VGP of the combined data set of normal and reversed polarities is located at $87.5^{\circ} \mathrm{N}$ and $4.0^{\circ} \mathrm{W}\left(\mathrm{A}_{95}=2.3^{\circ}\right)$, which is close to the geographic pole. The angular standard deviation (ASD) around the mean VGP resulted in $14.6^{\circ}\left(+1.3^{\circ}\right.$, $-1.2^{\circ}$ ). There is no significant inclination anomaly for the time-averaged field. However, if the plate motion is not taken into account, a significant anomaly of $-3.4^{\circ}$ would be observed at a $95 \%$ confidence level. This suggests that correction for the absolute plate motion is needed for the precise paleodirectional analysis of the Pacific region. These features are also supported by an analysis combining the previous data set with our data.
\end{abstract}

\section{Introduction}

Paleodirectional studies are quite important for finding the paleosecular variation (PSV) and time-averaged features of a geomagnetic field. Over the last decade, several paleomagnetic studies have proposed inclusive models for the past few million years, (e.g. Gubbins and Kelly, 1993; Johnson and Constable, 1995, 1997; Kelly and Gubbins, 1997; Kono et al., 2000). A common conclusion from these models is that the time-averaged field (TAF) is dominated by a dipole field and is associated with a certain number of non-dipole fields. Because of non-dipole components, there is a latitudinal dependence in the angular standard deviation (ASD) of the virtual geomagnetic poles (VGPs), as pointed out by Cox (1970). Non-dipole fields may also generate systematic inclination offsets from the geocentric axial dipole (GAD) field, that is an inclination anomaly. McElhinny et al. (1996a) suggested that the inclination anomaly for the past $5 \mathrm{Ma}$ TAF was attributed to the zonal quadrupole and octupole.

Although a reliable and global database is required for these studies, paleodirections have scarcely been provided from the Southern Hemisphere. As a comprehensive database covering the past $5 \mathrm{Ma}$, Johnson and Constable (1996) compiled 1610 independent paleodirectional data

*Present address: Geological Survey of Japan, AIST, Tsukuba Central 7, Tsukuba, Ibaraki 305-8567, Japan.

Copy right(c) The Society of Geomagnetism and Earth, Planetary and Space Sciences (SGEPSS); The Seismological Society of Japan; The Volcanological Society of Japan; The Geodetic Society of Japan; The Japanese Society for Planetary Sciences. from the Northern Hemisphere but only 590 from the Southern. Hence, hotspot basalts in the Southern Hemisphere are good candidates for improving this situation. This is because they are expected to have stable remanences and frequent eruptions can yield detailed paleomagnetic records.

The Society Islands in French Polynesia are composed mainly of 0-5 Ma hotspot basalts. Duncan (1975) paleomagnetically studied volcanic rocks from five islands of Tahiti, Moorea, Huahine, Raiatea and Borabora. He retrieved 35 normal, 18 reversed and six intermediate polarities, applying a threshold VGP latitude of $50^{\circ}$. However, these data may be of insufficient quality for modern paleosecular variation studies because (1) the directions were not determined by the principal component analysis (Kirschvink, 1980) and (2) some of their magnetic cleanings do not seem to be sufficient. Especially concerning the latter point, 59 directions were obtained with magnetic cleanings up to $20 \mathrm{mT}$, and six of them were determined from non-cleaning remanences. Roperch and Duncan (1990) reported transient geomagnetic fields from Huahine, while Chauvin et al. (1990) observed 35 normal, 41 reversed, and 47 intermediate directions from 123 sites in Tahiti Nui. The time span was, however, relatively short, since they were mainly interested in the transitional behavior of the geomagnetic field.

In this study, volcanic rocks have been paleomagnetically measured for 154 sites at Maupiti, Borabora, Tahaa, Raiatea, Huahine, Moorea, and Tahiti by both thermal and AF demagnetizations in order to extract primary remanences. Based on these results, we will discuss the precise PSV and TAF for the past $5 \mathrm{Ma}$ in the Society Islands. 


\section{Samples and Plate Motion}

The Society Islands consist of 10 volcanic islands and several seamounts. They are hotspot origin and show a chain-like configuration in a northwest-southeast direction delineating the Pacific plate motion. The present hotspot is located at $18^{\circ} \mathrm{S}, 148^{\circ} \mathrm{W}$ at the southeastern end of the archipelago (Gripp and Gordon, 1990), where several active submarine volcanoes are observed. The basaltic volcanism began about $5 \mathrm{Ma}$ (Duncan and McDougall, 1976). The samples were collected from 101 sites of lava sequences and from 53 sites of dikes and single lavas, mostly by a portable engine drill. The sample orientation was carried out by a magnetic compass and/or a sun compass. As shown in Fig. 1, the sampling sites are distributed at Maupiti (24 sites), Borabora (21), Tahaa (20), Raiatea (24), Huahine (21), Moorea (33) and Tahiti (11).

The K-Ar ages reported in the literature can be summarized as 3.9-4.51 Ma for Maupiti, 3.10-3.45 Ma for BoraBora, 2.8-3.2 Ma for Tahaa, 2.44-2.75 Ma for Raiatea, 2.03.08 Ma for Huahine, 1.36-1.72 Ma for Moorea, and 0.621.19 Ma for Tahiti (Blais et al., 1997, 2000; Chauvin et al.,

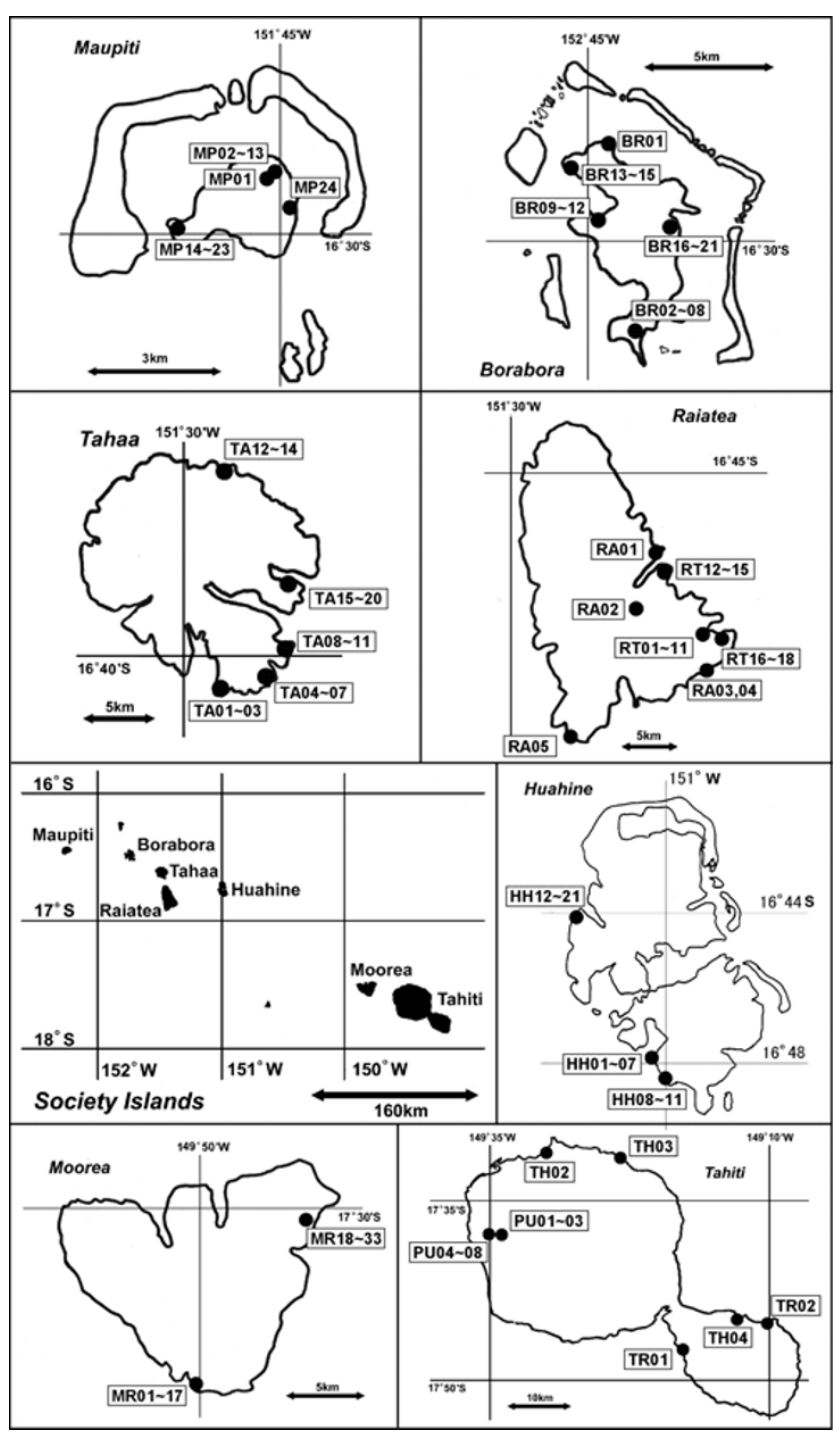

Fig. 1. A map showing the sampling sites.
1990; Duncan and McDougall, 1976; Guillou et al., 1998; Roperch and Duncan, 1990; Singer et al., 1999). Therefore, these islands provide paleomagnetic record of the past 5 Ma. However, it should be noticed that the absolute motion of the Pacific plate evokes a few degrees of change in the sampling site positions over a few million years. According to the HS2-NUVEL1 model (Gripp and Gordon, 1990), the Pacific-plate absolute motion has Euler pole at $60.2^{\circ} \mathrm{S}$, $90.0^{\circ} \mathrm{E}$ and an angular speed of $0.98^{\circ} / \mathrm{Ma}$. Applying this model, the original positions are gathered and make a cluster at a mean position of $18.0^{\circ} \mathrm{S}, 148.6^{\circ} \mathrm{W}$ with a $95 \%$ confidence circle of $0.2^{\circ}$, which is close to the present hotspot. The correction for the Pacific-plate motion is not negligible for precise paleodirectional analysis, because inclination of the GAD field changes from $-33.0^{\circ}$ at $18^{\circ} \mathrm{S}$ to $-31.4^{\circ}$ at $17^{\circ} \mathrm{S}$. Therefore, we will compare the data with and without the plate-motion correction later.

\section{Experimental Procedures and Demagnetization Results}

More than three specimens from each site were subjected to stepwise thermal demagnetization up to $600^{\circ} \mathrm{C}$ in the air at $20-50^{\circ} \mathrm{C}$ intervals. Alternating field (AF) demagnetization was also performed on other specimens at 5 or $10 \mathrm{mT}$ intervals up to a $160 \mathrm{mT}$ peak field. All the natural remanent magnetizations (NRMs) were measured by a spinner magnetometer (Natsuhara-Giken SMD-88 and DSPIN-2; Kono et al., 1984, Kono et al., 1997). All paleodirections were determined by principal-component analysis (Kirschvink, 1980). We measured 885 paleomagnetic cores from 154 sites and their NRM intensities ranged from $1.29 \times 10^{-4}$ to $1.50 \times 10^{-2}$ $\mathrm{Am}^{2} / \mathrm{kg}$ with a mode around $1 \times 10^{-3} \mathrm{Am}^{2} / \mathrm{kg}$.

Primary remanences were easily isolated from 140 sites using both thermal and AF demagnetizations (Fig. 2), yielding site mean paleodirections with $\alpha_{95} \leq 15^{\circ}$. Among them,

\section{(A)}
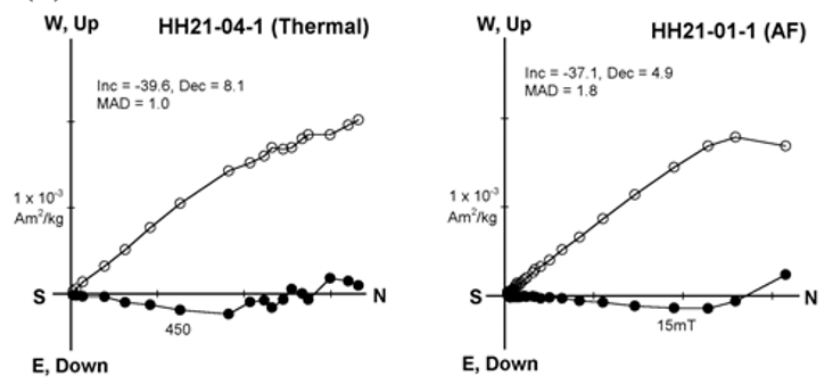

(B)
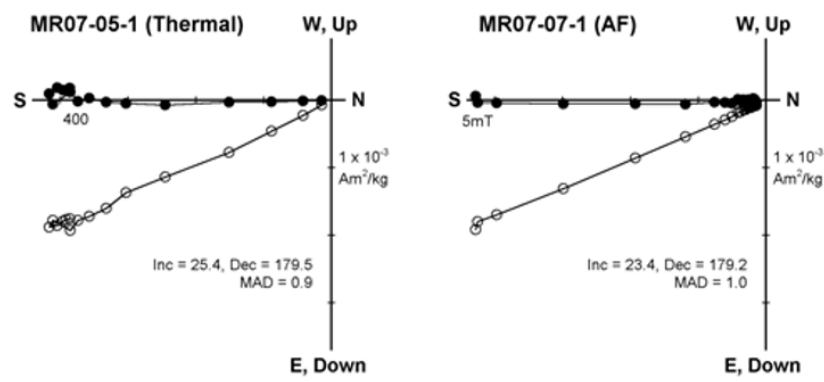

Fig. 2. Representative stepwise thermal and AF demagnetization diagrams for (A) normal and (B) reversed directions. 
Table 1. Paleodirections and VGPs for the dikes and single lavas. Site, Site ID; $n / N$, number of the specimens used for calculation and demagnetization; $\alpha_{95}$, a 95\% confidence circle; PLat, PLong, latitude and longitude of VGP. The prime $\left({ }^{\prime}\right)$ denotes the values after plate-motion correction. Precise site localities are referred to in Fig. 1.

\begin{tabular}{|c|c|c|c|c|c|c|c|c|}
\hline Site & $\overline{\mathrm{Dec}}$ & Inc & $n / N$ & $\overline{\alpha_{95}}$ & PLat & PLong & $\overline{\text { PLat' }^{\prime}}$ & $\overline{\mathrm{PLO}}$ \\
\hline \multicolumn{9}{|c|}{ Maupiti $\left(16.44 \sim 16.45^{\circ} \mathrm{S}, 152.25 \sim 152.28^{\circ} \mathrm{W}\right)$} \\
\hline MP01 & -80.4 & -79.7 & $5 / 5$ & 4.3 & 18.7 & 48.6 & 20.0 & 52.8 \\
\hline MP14 & 25.4 & -50.8 & $5 / 5$ & 5 & 62.5 & -24.5 & 64.4 & -22.6 \\
\hline MP15 & 22.1 & -54.9 & $5 / 5$ & 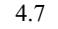 & 62.7 & -14.2 & 64.7 & -11.5 \\
\hline MP16 & 17.3 & -7.2 & $5 / 5$ & 7 & 68.7 & -97.4 & 68.4 & -99.0 \\
\hline MP17 & 8.0 & -29.3 & $3 / 6$ & 20.2 & - & - & - & - \\
\hline MP18 & 4.7 & -40.4 & $5 / 5$ & 70 & 82.0 & -5.3 & 84.1 & -3.0 \\
\hline MP19 & -3.0 & -36.8 & $5 / 5$ & 3. & 85.0 & 62. & 85.5 & 90.0 \\
\hline MP20 & -3.5 & -49.6 & $5 / 5$ & & 75.6 & 39.9 & 77.1 & 49.5 \\
\hline MP21 & -9.7 & -18.0 & $5 / 5$ & 4.2 & 78.1 & 153.9 & 76.2 & 160.9 \\
\hline MP22 & - & - & $0 / 7$ & - & - & - & - & - \\
\hline MP23 & 0.1 & -25.3 & $5 / 5$ & 4.0 & 86.8 & -151.3 & 85.0 & -159.6 \\
\hline MP24 & 4.5 & -38.6 & $5 / 5$ & 9.2 & 83.2 & -10 . & 85.2 & -10.7 \\
\hline \multicolumn{9}{|c|}{ Borabora $\left(16.46 \sim 16.54^{\circ} \mathrm{S}, 151.73 \sim 151.76^{\circ} \mathrm{W}\right)$} \\
\hline BR01 & -157.2 & 43.2 & $4 / 7$ & 12.5 & -67.1 & 144.3 & -68.4 & 144.8 \\
\hline BR02 & -8.8 & -31.7 & $7 / 7$ & & 81.6 & 112. & 80.8 & 124.6 \\
\hline $\mathrm{BI}$ & -4.0 & -28.9 & $5 / 5$ & & 86.0 & 153. & 84.7 & 148.9 \\
\hline BR08 & -3.7 & -53.4 & $7 / 7$ & 2. & 72.3 & 38.4 & 73.5 & 44.6 \\
\hline BR09 & 2.8 & -41.9 & $4 / 8$ & 5 & 81.9 & 9.5 & 83.5 & 14.9 \\
\hline BR10 & 17.6 & -13.1 & $6 / 6$ & 3. & 70.2 & -89.5 & 70.2 & -91.1 \\
\hline $\mathrm{BI}$ & 20.5 & -37 & $7 / 7$ & & 70.1 & -4 & 71.2 & -46.4 \\
\hline BR12 & 3.3 & -21.1 & $6 / 8$ & & 83.6 & -121.3 & 82.6 & -129.3 \\
\hline BR13 & 147.4 & 23.3 & $4 / 7$ & & -58.2 & 114.8 & -58.9 & 115.3 \\
\hline BR16 & 16.9 & -39.0 & $7 / 7$ & 4 & 73.1 & -40 & 74.3 & -40.7 \\
\hline BR17 & 7.4 & -49.7 & $5 / 5$ & 14.4 & 61.4 & -27.5 & 62.8 & -26.1 \\
\hline \multicolumn{9}{|c|}{ Tahaa $\left(16.58 \sim 16.59^{\circ} \mathrm{S}, 151.48 \sim 151.49^{\circ} \mathrm{W}\right)$} \\
\hline $\mathrm{TA}$ & 6.3 & 43.9 & $5 / 5$ & & -63.9 & 143.4 & -65.1 & 144.2 \\
\hline TA13 & 8.6 & 37.3 & $9 / 10$ & 0 & -69.3 & -76.6 & -68.9 & -70.4 \\
\hline TA14 & 203.0 & 76.0 & $5 / 5$ & 68.7 & - & - & - & - \\
\hline \multicolumn{9}{|c|}{ Raiatea $\left(16.80 \sim 16.92^{\circ} \mathrm{S}, 151.37 \sim 151.46^{\circ} \mathrm{W}\right)$} \\
\hline RT0 & 4.6 & -39.3 & $7 / 7$ & 32 & 83.1 & -9.2 & 84.3 & -8.8 \\
\hline RT02 & 7.6 & -42.4 & $6 /$ & 4. & 79.5 & -12 & 80.8 & -12.0 \\
\hline RT03 & .3 & -26.4 & 6 & & 86.8 & -12 & 86.0 & -140.1 \\
\hline & & -42 & 5 & & 3.5 & -16.3 & 79.8 & -15.9 \\
\hline RT09 & & 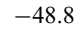 & $5 /$ & & 76.1 & 7.0 & 77.3 & 10.1 \\
\hline RA01 & 6.0 & -24.7 & $4 / 4$ & 3.2 & 83.0 & -94.1 & 82.8 & -102.0 \\
\hline 00 & - & - & $0 / 3$ & - & - & - & - & - \\
\hline & & 9.2 & & - & - & - & - & - \\
\hline & -3.5 & -23.9 & $1 /$ & - & - & - & - & - \\
\hline 104 & 2.4 & -19.1 & $1 /$ & - & - & - & - & - \\
\hline RA05 & -2.3 & -36.2 & $4 / 4$ & 14.7 & 86.1 & 62.6 & 86.5 & 83.7 \\
\hline \multicolumn{9}{|c|}{ Huahine $\left(16.74 \sim 16.81^{\circ} \mathrm{S}, 151.00 \sim 151.04^{\circ} \mathrm{W}\right)$} \\
\hline $\mathrm{H}$ & -1.2 & -31.3 & $7 / 7$ & 44 & 88.8 & 111.9 & 88.1 & 146.5 \\
\hline & 0. & -48 & 1 & & 77.6 & 25.3 & 78.6 & 29.7 \\
\hline & -1.5 & -28 & $6 / 7$ & 5 & 87.7 & 170. & 86.6 & 174.9 \\
\hline & 49 & -26 & 6 & & 84.4 & -9 & 84.3 & -100.6 \\
\hline & & -13 & 6 & & & -9 & 71 & -91.7 \\
\hline & & & 5 & & 56.0 & -133.5 & 55.2 & -132.8 \\
\hline HH14 & 24.6 & -21.5 & $5 / 5$ & 10.6 & 65.5 & -71.0 & 65.9 & -71.3 \\
\hline HH15 & 26.2 & 20.7 & $5 / 5$ & 30.6 & - & - & - & - \\
\hline \multicolumn{9}{|c|}{ Tahiti $\left(17.52 \sim 17.78^{\circ} \mathrm{S}, 149.17 \sim 149.59^{\circ} \mathrm{W}\right)$} \\
\hline & & -62.3 & $6 / 7$ & 4.1 & 58.9 & 1.4 & 59.3 & 2.2 \\
\hline PU( & 19.9 & -33.4 & 111 & 0 & 71.1 & -54.7 & 71.4 & -55.0 \\
\hline PU06 & -174.2 & 32.1 & $10 / 10$ & 5 & -84.5 & 118.9 & -84.7 & 155.6 \\
\hline & -1.0 & -45.2 & $8 / 10$ & 11. & & 36. & 81.3 & 38.7 \\
\hline & -8.0 & 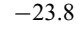 & 0 & 5 & 80.6 & 154.6 & 80.2 & 156.4 \\
\hline & 12.5 & -29.4 & $4 / 4$ & 4.7 & 77.9 & -66.3 & 78.1 & -67.5 \\
\hline $\mathrm{THO}$ & 4.7 & -18.3 & $4 / 4$ & 7.7 & 80.7 & -119.6 & 80.5 & -121.2 \\
\hline TH04 & 7.4 & -18.6 & $4 / 4$ & 3.7 & 79.1 & -106.9 & 79.0 & -108.3 \\
\hline
\end{tabular}

130 out of 140 directions satisfy $\alpha_{95} \leq 10^{\circ}$. These samples have stable and relatively strong magnetizations of $\sim 10^{-3}$ $\mathrm{Am}^{2} / \mathrm{kg}$ with high blocking temperatures and coercivities. Their secondary components were efficiently removed by $350-450^{\circ} \mathrm{C}$ heating or by $10-20 \mathrm{mT}$ AF cleaning. Most of their MAD values in the principal-component analysis were less than $5.0^{\circ}$.

On the other hand, primary components were extracted, but the internal consistency was not $\operatorname{good}\left(\alpha_{95}>15^{\circ}\right)$ for six sites. The magnetizations of the other six sites were too weak $\left(\sim 10^{-4} \mathrm{Am}^{2} / \mathrm{kg}\right)$ to extract stable components. Since we did not collect enough cores from the two sites of RA02b and RA03 in Raiatea, they gave only reference directions. Therefore, results from these 14 sites were not incorporated into the later statistical analyses.
Table 2. Paleodirections and VGPs for the lava sequences at Maupiti, Borabora, Tahaa, and Raiatea. Results are listed in sequential order (bottom is the older). Precise site localities are referred to in Fig. 1.

\begin{tabular}{|c|c|c|c|c|c|c|c|c|}
\hline Site & Dec & Inc & $n / N$ & $\alpha_{95}$ & PLat & PLong & PLat' $^{\prime}$ & PLong' $^{\prime}$ \\
\hline \multicolumn{9}{|c|}{ Maupiti } \\
\hline MP13 & -3.8 & -20.2 & $5 / 5$ & 5.3 & 82.9 & 175.5 & 80.9 & 179.7 \\
\hline MP12 & -4.6 & -20.1 & $5 / 5$ & 5.8 & 82.4 & 170.6 & 80.4 & 175.8 \\
\hline MP11 & 2.4 & -24.5 & $5 / 5$ & 3.6 & 85.7 & -118.5 & 84.5 & -134.2 \\
\hline MP10 & 0.3 & -29.4 & $5 / 5$ & 7.9 & 89.2 & -129.9 & 87.4 & -164.6 \\
\hline MP09 & -3.0 & -29.4 & $5 / 5$ & 2.9 & 87.1 & 130.8 & 85.4 & 153.5 \\
\hline MP08 & -5.5 & -27.8 & $5 / 5$ & 3.8 & 84.5 & 134.2 & 82.8 & 149.2 \\
\hline MP07 & -4.8 & -25.3 & $5 / 5$ & 7.7 & 84.4 & 151.1 & 82.5 & 161.8 \\
\hline MP06 & -2.0 & -27.6 & $5 / 5$ & 6.5 & 87.4 & 161.2 & 85.4 & 172.2 \\
\hline MP05 & -1.0 & -25.6 & $5 / 5$ & 2.3 & 86.9 & -169.8 & 84.9 & -171.0 \\
\hline MP04 & -4.7 & -28.0 & $5 / 5$ & 2.5 & 85.2 & 136.1 & 83.6 & 152.0 \\
\hline MP03 & 0.0 & -25.3 & $5 / 5$ & 2.4 & 86.9 & -153.0 & 85.0 & -160.7 \\
\hline MP02 & 3.3 & -25.0 & $5 / 5$ & 3.7 & 85.4 & -107.9 & 84.4 & -125.0 \\
\hline \multicolumn{9}{|c|}{$\begin{array}{l}\text { Borabora } \\
\left.B R-A\left(16.54^{\circ} \mathrm{S}, 151.73^{\circ} \mathrm{W}\right)\right\rangle\end{array}$} \\
\hline BR07 & -176.9 & 24.6 & $7 / 7$ & 4.2 & -85.3 & 67.6 & -84.5 & 54.7 \\
\hline BR04 & -160.4 & 29.6 & $7 / 7$ & 4.2 & -71.2 & 118.9 & -71.9 & 117.5 \\
\hline BR05 & -159.1 & 26.7 & $5 / 5$ & 2.4 & -69.7 & 114.2 & -70.3 & 113.0 \\
\hline BR06 & -170.6 & 28.9 & $5 / 5$ & 3.0 & -80.9 & 112.5 & -81.4 & 105.6 \\
\hline \multicolumn{9}{|c|}{$\left\langle\right.$ Sequence BR-B $\left.\left(16.47^{\circ} \mathrm{S}, 151.76^{\circ} \mathrm{W}\right)\right\rangle$} \\
\hline BR15 & -159.2 & 42.2 & $7 / 7$ & 2.8 & -69.0 & 143.9 & -70.3 & 144.1 \\
\hline BR14 & -160.2 & 37.6 & $5 / 6$ & 3.1 & -70.7 & 135.1 & -71.9 & 134.5 \\
\hline \multicolumn{9}{|c|}{$\left\langle\right.$ Sequence BR-C $\left.\left(16.50^{\circ} \mathrm{S}, 151.73^{\circ} \mathrm{W}\right)\right\rangle$} \\
\hline BR21 & 11.5 & -39.4 & $5 / 5$ & 6.5 & 77.6 & -31.6 & 79.0 & -32.9 \\
\hline BR20 & 6.4 & -40.6 & $5 / 5$ & 5.4 & 81.0 & -12.8 & 82.6 & -12.4 \\
\hline BR19 & 7.8 & -41.3 & $5 / 5$ & 5.3 & 79.8 & -15.9 & 81.3 & -15.7 \\
\hline BR18 & 15.0 & -45.8 & $8 / 8$ & 3.2 & 72.4 & -21.5 & 73.9 & -20.6 \\
\hline \multicolumn{9}{|c|}{$\begin{array}{l}\text { Tahaa } \\
\left.4\left(16.68^{\circ} \mathrm{S}, 151.48^{\circ} \mathrm{W}\right)\right\rangle\end{array}$} \\
\hline TA03 & 131.6 & 13.1 & $5 / 5$ & 13.5 & -41.7 & -55.4 & -40.8 & -51.9 \\
\hline TA02 & 124.1 & 5.8 & $4 / 9$ & 61.5 & - & - & - & - \\
\hline TA01 & 165.0 & -8.1 & $7 / 7$ & 16.4 & - & - & - & \\
\hline \multicolumn{9}{|c|}{$\left\langle\right.$ Sequence TA-B $\left.\left(16.68^{\circ} \mathrm{S}, 151.45^{\circ} \mathrm{W}\right)\right\rangle$} \\
\hline TA07 & -67.4 & 70.3 & $5 / 5$ & 5.3 & -1.1 & 176.0 & -2.5 & 178.6 \\
\hline TA06 & -44.0 & 75.6 & $5 / 5$ & 11.0 & 3.4 & -170.0 & 2.0 & -167.5 \\
\hline TA05 & -43.6 & 78.5 & $7 / 7$ & 4.2 & -0.2 & -166.6 & -1.6 & -164.0 \\
\hline TA04 & -14.4 & 68.5 & $4 / 5$ & 11.8 & 20.4 & -160.9 & 19.1 & -158.5 \\
\hline \multicolumn{9}{|c|}{$\left\langle\right.$ Sequence TA-C $\left.\left(16.66^{\circ} \mathrm{S}, 151.44^{\circ} \mathrm{W}\right)\right\rangle$} \\
\hline TA11 & 173.4 & 22.6 & 7/7 & 12.4 & -81.9 & -25.1 & -80.6 & -18.9 \\
\hline TA10 & -173.6 & 21.4 & $5 / 5$ & 6.9 & -81.6 & 77.5 & -81.2 & 70.7 \\
\hline TA09 & -177.8 & 20.5 & $5 / 5$ & 3.3 & -83.6 & 47.9 & -82.5 & 42.0 \\
\hline TA08 & 179.1 & 17.8 & $7 / 7$ & 7.5 & -82.4 & 21.7 & -81.1 & 20.6 \\
\hline \multicolumn{9}{|c|}{$\left\langle\right.$ Sequence TA-D $\left.\left(16.64^{\circ} \mathrm{S}, 151.44^{\circ} \mathrm{W}\right)\right\rangle$} \\
\hline TA20 & -16.8 & -55.5 & $5 / 5$ & 5.4 & 65.6 & 62.9 & 66.2 & 68.4 \\
\hline TA19 & -12.9 & -54.3 & $5 / 5$ & 9.2 & 68.5 & 58.4 & 69.2 & 64.2 \\
\hline TA18 & -26.5 & -50.8 & $6 / 6$ & 3.7 & 61.7 & 82.0 & 61.8 & 87.2 \\
\hline TA17 & -16.8 & -59.0 & $7 / 7$ & 5.9 & 62.6 & 57.4 & 63.4 & 62.5 \\
\hline TA16 & -5.9 & -60.9 & $5 / 7$ & 7.7 & 64.2 & 38.7 & 65 & 43.3 \\
\hline TA15 & -23.0 & -49.2 & $7 / 7$ & 2.0 & 65.1 & 81.8 & 65.2 & 87.5 \\
\hline \multicolumn{9}{|c|}{$\left\langle\right.$ Sequence RT-A $\left.\left(16.85^{\circ} \mathrm{S}, 151.37^{\circ} \mathrm{W}\right)\right\rangle$} \\
\hline RT04 & -20.0 & 52.5 & $6 / 7$ & 42.3 & the & 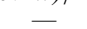 & - & - \\
\hline RT05 & 2.7 & 51.7 & $6 / 7$ & 3.0 & 40.7 & -148.4 & 39.6 & -146.8 \\
\hline RT06 & -4.5 & 59.9 & $5 / 5$ & 4.8 & 32.3 & -155.4 & 31.1 & -153.5 \\
\hline RT07 & -47.3 & 67.8 & $3 / 7$ & 2.8 & 10.7 & -179.6 & 9.5 & -177.4 \\
\hline RT10 & -41.4 & -28.3 & $7 / 7$ & 9.3 & 50.2 & 115.2 & 49.6 & 118.7 \\
\hline RT11 & -39.2 & -24.7 & $5 / 5$ & 8.8 & 52.0 & 119.1 & 51.4 & 122.6 \\
\hline \multicolumn{9}{|c|}{$\left\langle\right.$ Sequence RT-B $\left.\left(16.81^{\circ} \mathrm{S}, 151.39^{\circ} \mathrm{W}\right)\right\rangle$} \\
\hline RT15 & 4.3 & -35.9 & $6 / 7$ & 2.9 & 84.9 & -23.5 & 86.0 & -28.3 \\
\hline RT14 & 1.3 & -35.2 & $5 / 5$ & 2.4 & 87.1 & 3.2 & 88.3 & 8.7 \\
\hline RT13 & 5.0 & -39.2 & $5 / 5$ & 4.2 & 82.8 & -12.2 & 84.1 & -12.4 \\
\hline RT12 & 7.3 & -39.2 & $7 / 7$ & 2.0 & 81.3 & -22.3 & 82.4 & -23.6 \\
\hline \multicolumn{9}{|c|}{$\left\langle\right.$ Sequence RT-C $\left.\left(16.85^{\circ} \mathrm{S}, 151.36^{\circ} \mathrm{W}\right)\right\rangle$} \\
\hline RT16 & 6.1 & -34.7 & $5 / 5$ & 8.5 & 83.7 & -39.1 & 84.7 & -45.3 \\
\hline RT17 & 5.8 & -36.4 & $5 / 6$ & 13.3 & 83.5 & -28.6 & 84.6 & -32.6 \\
\hline RT18 & 14.3 & -24.7 & $6 / 7$ & 4.9 & 75.6 & -75.2 & 75.9 & -77.9 \\
\hline
\end{tabular}

All the measurement results are summarized in Table 1 for the dikes and single lavas, and in Tables 2 and 3 for the lava sequences. The VGP positions were calculated for 140 sites with $\alpha_{95} \leq 15^{\circ}$. The correction of the sampling localities for the absolute motion of the Pacific plate was applied in the VGP calculations.

\section{Discussion}

We take a threshold VGP latitude of $50^{\circ}$ in the definition of polarity. In other words, the VGP latitude of normal or reversed polarity is higher than $50^{\circ}$, and the intermediate sites 
Table 3. Paleodirections and VGPs for the lava sequences in Huahine, Moorea, and Tahiti.

\begin{tabular}{|c|c|c|c|c|c|c|c|c|}
\hline Site & $\overline{\text { Dec }}$ & Inc & $n / N$ & $\alpha_{95}$ & PLat & PLong & PLat' $^{\prime}$ & PLong' $^{\prime}$ \\
\hline \multicolumn{9}{|c|}{$\begin{array}{c}\text { Huahine } \\
\left\langle\text { Sequence } \mathrm{HH}-\mathrm{A}\left(16.80^{\circ} \mathrm{S}, 151.01^{\circ} \mathrm{W}\right)\right\rangle\end{array}$} \\
\hline HH03 & 4.7 & \multicolumn{5}{|c|}{$\left\langle\right.$ Sequence $\left.\mathrm{HH}-\mathrm{A}\left(16.80^{\circ} \mathrm{S}, 151.01^{\circ} \mathrm{W}\right)\right\rangle$} & 80.2 & -124.3 \\
\hline HH02 & 6.6 & -20.8 & $8 / 8$ & 1.8 & 81.2 & -103.3 & 80.9 & -108.1 \\
\hline HH01 & 3.1 & -22.8 & $7 / 7$ & 1.8 & 84.2 & -119.0 & 83.6 & -125.9 \\
\hline HH04 & 6.2 & -21.2 & $5 / 5$ & 2.0 & 81.6 & -10 & 81.3 & -109.6 \\
\hline \multicolumn{9}{|c|}{$\left\langle\right.$ Sequence $\left.H H-B\left(16.81^{\circ} \mathrm{S}, 151.00^{\circ} \mathrm{W}\right)\right\rangle$} \\
\hline HH11 & 7.0 & -26.8 & $6 / 6$ & 4.4 & 82.7 & -81.3 & 82.8 & -88.1 \\
\hline HH10 & 20.0 & -34.3 & $6 / 6$ & 4.7 & 70.8 & -51.9 & 71.5 & -52.6 \\
\hline HH09 & 22.3 & -31.1 & 7/7 & 3.4 & 68.7 & -57.8 & 69.3 & -58.4 \\
\hline \multicolumn{9}{|c|}{$\left\langle\right.$ Sequence $\left.\mathrm{HH}-\mathrm{C}\left(16.74^{\circ} \mathrm{S}, 151.04^{\circ} \mathrm{W}\right)\right\rangle$} \\
\hline HH16 & 9.9 & -34.6 & $7 / 7$ & 2.1 & 80.3 & -45.8 & 81.1 & -48.8 \\
\hline HH17 & 6.7 & -40.8 & $5 / 5$ & 2.9 & 80.9 & -13.6 & 81.9 & -13.4 \\
\hline HH18 & 7.4 & -38.1 & $5 / 5$ & 4. & 81.6 & -26.4 & 82.6 & -28.1 \\
\hline $\mathrm{HH} 20$ & 1.3 & -40 . & $7 / 7$ & 1. & 83.3 & 18.7 & 84.3 & 24.4 \\
\hline $\mathrm{H}$ & 2.1 & -39.1 & $5 / 5$ & 2.3 & 84.2 & 9.0 & 5.3 & 13.3 \\
\hline $\mathrm{HH} 21$ & 4.8 & -37.5 & $5 / 5$ & 1.9 & 83.8 & -17.2 & 84.9 & -18.8 \\
\hline \multirow{2}{*}{\multicolumn{9}{|c|}{ Moorea }} \\
\hline & & & & & & & & \\
\hline R01 & 0.4 & 31.0 & $7 / 7$ & 2.5 & -80.8 & -56.0 & -80.3 & -51.1 \\
\hline MR02 & 172.6 & 29.5 & $5 / 5$ & 3.8 & -82.6 & -46.6 & -82.1 & -41.4 \\
\hline MR03 & 171.6 & 36.1 & $5 / 5$ & 2.4 & -81.7 & -78.2 & -81.5 & -72.0 \\
\hline 4 & & 3 & $5 / 5$ & 0. & -81.9 & -88.3 & -81.8 & -81.7 \\
\hline MR05 & 173.7 & 32 & $5 / 5$ & 2. & -84.0 & -61.6 & -83.6 & -54.4 \\
\hline MR06 & 173.5 & 30 & $7 / 7$ & 4.8 & -83.7 & -50.0 & -83.2 & -43.9 \\
\hline MR07 & 179.5 & 24.3 & $5 / 5$ & 3.2 & -85.1 & 25.0 & -84.4 & 23.0 \\
\hline & & - & $4 / 4$ & 4. & -83.1 & -49.4 & -82.6 & -43.8 \\
\hline 10 & & 2 & $5 /$ & 4 & -74.4 & -66 & -74.0 & -6 \\
\hline MR10 & 1 & & $5 / 5$ & 3. & -80.3 & -37 & -79.7 & -34.1 \\
\hline MR11 & 172.8 & 32.5 & $5 / 5$ & 2.1 & -83.2 & -61.5 & -82.8 & -55.0 \\
\hline MR12 & 174.9 & 293 & $5 / 5$ & 3 & -84.7 & -3 & -84.1 & -3 \\
\hline & & & $5 / 5$ & & -81.6 & -3 & -8 & -2 \\
\hline M & & & $5 / 5$ & 4. & -82.9 & -24.2 & -82.2 & -20.7 \\
\hline MR15 & -171.7 & 23.2 & $5 / 5$ & 5.7 & -80.3 & 86.9 & -80.2 & 83.8 \\
\hline MR16 & 174 & 32.8 & $7 / 7$ & 3.0 & -84.4 & -63.2 & -84.0 & -55.5 \\
\hline MR17 & 179.9 & 30.2 & $5 / 5$ & 7.8 & -88.6 & 26 & -87.9 & 18.4 \\
\hline \multicolumn{9}{|c|}{$\left\langle\right.$ Sequence MR-B $\left.\left(17.50^{\circ} \mathrm{S}, 149.77^{\circ} \mathrm{W}\right)\right\rangle$} \\
\hline MR18 & - & - & $0 / 7$ & - & - & - & - & - \\
\hline & -166.1 & 40.8 & $5 / 5$ & 5.7 & -75.7 & 146.6 & -76.3 & 146.2 \\
\hline & - & & 5 & & -72.8 & -158.2 & -7 & \\
\hline & 162 & 13 & $5 / 5$ & 1. & -70.2 & -29.4 & -69.5 & -27.2 \\
\hline & & 5 & $5 / 5$ & 4.4 & -67.9 & -123.6 & -68.3 & -120.7 \\
\hline & -178.2 & & $7 / 7$ & 6.0 & -64.9 & -153.0 & -65.6 & -150.9 \\
\hline & & & & & -69.2 & -12 & -6 & -119.5 \\
\hline & & & $5 / 6$ & 3 & -67.8 & -132.7 & -68.3 & -1 \\
\hline & & & $5 / 5$ & 3. & -68.4 & -122.5 & -68.8 & -119.6 \\
\hline & -163.0 & 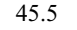 & $5 / 5$ & 4.2 & -71.7 & 154.3 & -72.4 & 154.6 \\
\hline & -175.8 & & $5 /$ & 3 & -86.0 & 115.4 & -86.3 & 106.3 \\
\hline & 176.7 & -0.2 & 5 & 3.8 & -72.1 & 19.5 & -71.4 & 20.0 \\
\hline & & - & $0 / 7$ & - & - & - & - & - \\
\hline & 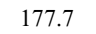 & 51.9 & $5 / 5$ & 3.4 & -74.8 & -142.3 & -75.4 & -139.2 \\
\hline & -174.3 & 68.0 & $7 / 7$ & 3.6 & -56.2 & -156.2 & -56.9 & -154.4 \\
\hline & 162.6 & 60.7 & $5 / 5$ & 5.2 & -61.6 & -121.8 & -62.0 & -119.3 \\
\hline \multirow{2}{*}{\multicolumn{9}{|c|}{$\left\langle\right.$ Sequence $\left.P U-A\left(17.63^{\circ} \mathrm{S}, 149.57^{\circ} \mathrm{W}\right)\right\rangle$}} \\
\hline & & & & & & & & \\
\hline PU0, & 162.1 & 35.6 & $3 / 6$ & 8.1 & -72.9 & -69.4 & -72.7 & -67.3 \\
\hline & & - & $0 / 6$ & - & - & - & - & - \\
\hline PU01 & 160.8 & 33.1 & $7 / 7$ & 4.6 & -71.8 & -63.9 & -71.6 & -61.9 \\
\hline
\end{tabular}

are for the VGP located between $50^{\circ} \mathrm{N}$ and $50^{\circ} \mathrm{S}$. Applying the plate-motion correction, 82 normal, 48 reversed, and 10 intermediate paleofields have been observed (Tables 1, 2 and 3). If an alternative threshold latitude of $45^{\circ}$ or $55^{\circ}$ is taken, one intermediate VGP (RT10) is changed to a normal one, or one normal VGP (RT11) is changed to an intermediate one. The overall feature is, however, unchanged so that the polarities in the present data set are considered to be robust against the selection of a threshold latitude of around $50^{\circ}$.

\subsection{VGP positions}

The resultant polarities are likely to be consistent with the geomagnetic polarity timescale of Cande and Kent (1995) on the basis of previous geochronological studies (see Section 2). All the VGP positions are illustrated in Fig. 3, where two sequences of intermediate VGPs are observed from the lava sequences of TA-B (TA04, TA05, TA06 and TA07) and RT-A (RT10, RT07, RT06 and RT05). These VGPs appear to

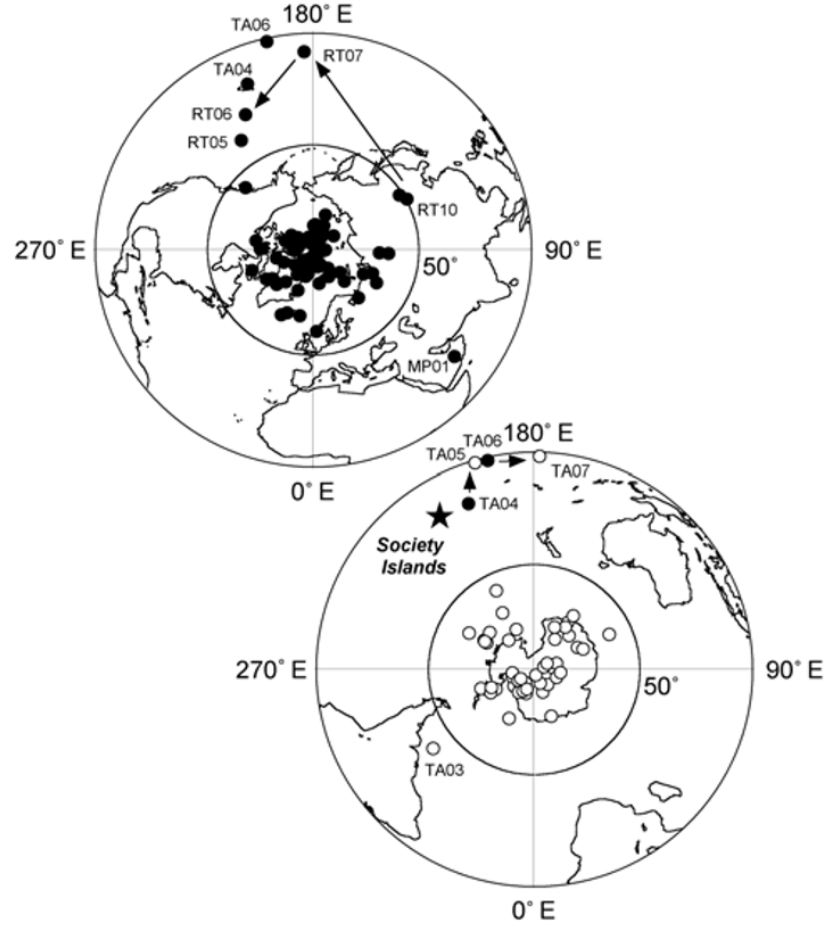

Fig. 3. Equal area projections of the VGP positions. Close and open circles are in the Northern and Southern Hemispheres, respectively. Star denotes the sampling sites. Arrows indicate the transitional VGP paths observed from the two lava sequences (TA-B and RT-A).

be clustered in the mid-Pacific region. This is quite different from the preferred longitudinal bands of the transitional VGP path (Laj et al., 1991) but this clustering shows similar behavior to that of the N-N excursion from Huahine (2.91-3.08 $\mathrm{Ma}$; Roperch and Duncan, 1990). Considering the formation ages of Tahaa (2.8-3.2 Ma) and Raiatea (2.44-2.75 Ma), the two sequences might record the same excursion, although a precise age determination will be necessary for more detailed comparison.

Excluding the intermediates, the VGP positions seem to be distributed around the geographic poles (Fig. 3). If we apply the Bingham statistics to the combined VGPs, the elongation parameter $\left(\alpha_{32} / \alpha_{31}\right.$; Tanaka, 2000) is calculated to be 1.15 . Since $\alpha_{32} / \alpha_{31}$ for the corresponding field directions results in 1.42, the VGP distribution is more Fisherian than that of the field directions. It is a similar behavior for the past 5 Ma Hawaiian data set (Tanaka, 2000), but this feature is less pronounced if the plate-motion correction is not taken into account; $\alpha_{32} / \alpha_{31}$ is 1.21 for the VGPs and 1.34 for the field directions. Therefore, the effect of absolute plate motion is not negligible for the precise analysis.

We also calculated mean VGPs for the normal, reversed, and combined data set (Table 4). The combined data set shows a small amount of offset from the geographic pole, but it is comparable with $\mathrm{A}_{95}$. If plate-motion correction is not applied, the offset becomes about $1^{\circ}$ larger than that with correction (Table 4). This suggests that the plate-motion correction reduces the offset of VGPs.

\subsection{Paleosecular variation}

As 95 out of 140 directions were obtained from the lava sequences (Tables 2 and 3), the serial correlation test (e.g. 
Table 4. Summary of mean VGP positions, ASD and inclination anomalies before and after plate-motion correction. $N$, number of data; Lat, Long, VGP latitude and longitude; A $_{95}, 95 \%$ confidence circle; ASD, angular standard deviation around the mean VGP and its $95 \%$ limits; $\Delta I$, inclination anomaly.

\begin{tabular}{|c|c|c|c|c|c|c|}
\hline & \multirow[t]{2}{*}{$N$} & \multicolumn{3}{|c|}{ mean VGP } & \multirow[t]{2}{*}{$\overline{\mathrm{ASD}}$} & \multirow[t]{2}{*}{$\Delta I$} \\
\hline & & Lat & Long & $\mathrm{A}_{95}$ & & \\
\hline \multicolumn{7}{|c|}{ before the plate motion correction } \\
\hline Normal & 83 & $85.8^{\circ}$ & $342.5^{\circ}$ & $2.9^{\circ}$ & $14.4^{\circ}\left(+1.8^{\circ},-1.4^{\circ}\right)$ & \\
\hline Reversed & 48 & $-86.4^{\circ}$ & $215.6^{\circ}$ & $4.1^{\circ}$ & $15.7^{\circ}\left(+2.5^{\circ},-1.9^{\circ}\right)$ & \\
\hline Combined & 131 & $86.4^{\circ}$ & $359.4^{\circ}$ & $2.3^{\circ}$ & $14.9^{\circ}\left(+1.4^{\circ},-1.1^{\circ}\right)$ & $-3.4^{\circ}$ \\
\hline \multicolumn{7}{|c|}{ after the plate motion correction } \\
\hline Normal & 82 & $86.8^{\circ}$ & $331.4^{\circ}$ & $2.8^{\circ}$ & $13.8^{\circ}\left(+1.7^{\circ},-1.3^{\circ}\right)$ & \\
\hline Reversed & 48 & $-87.1^{\circ}$ & $227.8^{\circ}$ & $4.1^{\circ}$ & $15.6^{\circ}\left(+2.6^{\circ},-1.9^{\circ}\right)$ & \\
\hline Combined & 130 & $87.5^{\circ}$ & $356.0^{\circ}$ & $2.3^{\circ}$ & $14.6^{\circ}\left(+1.3^{\circ},-1.2^{\circ}\right)$ & $-1.7^{\circ}$ \\
\hline
\end{tabular}

McElhinny et al., 1996b) may conceivably be applied. However, Love (1998) showed that directional changes of a few degrees were occasionally accompanied by intensity changes of tens of microteslas. He concluded that grouping based on directional similarity was quite risky. In this study, we tentatively treat all the measured directions as independent ones. The ASD for the combined VGPs around the mean $(N=130)$ was calculated by subtracting within-site angular dispersion $\left(S_{W}=5.4^{\circ}\right)$ from total dispersion $\left(S_{T}=14.7^{\circ}\right)$. Since the resultant ASD $\left(S_{F}\right)$ of $14.6^{\circ}\left(+1.3^{\circ},-1.2^{\circ}\right)$ is almost the same as $S_{T}$, the errors of the site mean direction $\left(\alpha_{95}\right)$ do not seriously affect the present ASD.

This ASD is thought to be reliable for the past $5 \mathrm{Ma}$ PSV in the Society Islands, although the ASD is sometimes seriously dependent on a cut-off angle (e.g. Chauvin et al., 1990; Shibuya et al., 1995). This is because the polarities of the present data set is almost insensitive to a VGP cutoff angle of around $50^{\circ}$, as stated at the beginning of this section. Even if the alternative cut-off angles (VGP latitudes) are adopted, the ASDs result in $14.9^{\circ}$ and $14.2^{\circ}$ for cutoff angles of $45^{\circ}$ and $55^{\circ}$, respectively, which are not so different from $S_{F}$ of $14.6^{\circ}$. This estimation is slightly larger than the previous results of $13.8^{\circ}\left(+2.1^{\circ},-1.6^{\circ}\right)$ by Duncan (1975), $13.9^{\circ}\left(+1.7^{\circ},-1.2^{\circ}\right)$ by Chauvin et al. (1990), and the Model $\mathrm{G}$ for the past $5 \mathrm{Ma}$ (McFadden et al., 1988, 1991). However, they are statistically indistinguishable at a $95 \%$ confidence level.

\subsection{Time-averaged inclination}

Time-averaged inclination is calculated to be $-34.7^{\circ}$ $\left(\alpha_{95}=2.6^{\circ}\right)$ from the combined data set $(N=130)$ after plate-motion correction. Since the inclination of the GAD field corresponds to $-33.0^{\circ}$ for the mean corrected site latitude $\left(18.0^{\circ} \mathrm{S}\right)$, the time-averaged inclination does not show a significant anomaly at a $95 \%$ confidence level. However, the situation is changed if the plate motion is not taken into account. For example, the mean site latitude is calculated to be $16.9^{\circ} \mathrm{S}$ and the corresponding GAD inclination is $-31.3^{\circ}$. Despite the fact that one intermediate direction (RT10) is converted to a normal one $(N=131)$, the mean inclination is still preserved. As a result, an inclination anomaly of $-3.4^{\circ}$ would be observed at a statistically significant level (Table 4).

The above result demonstrates the importance of the correction for the absolute plate motion. As the usual analysis concerning the inclination anomaly requires a resolution of $1^{\circ}$, the plate-motion correction cannot be ignored, especially for the mid-Pacific region. This is because the Pacificplate motion has evoked an $\sim 2^{\circ}$ latitudinal change in sampling sites near the Equator over 5 million years, resulting in an $\sim 4^{\circ}$ inclination change of the GAD field. Johnson and Constable (1997) reported an inclination anomaly of $-8.2^{\circ}$ in the Hawaiian Islands for the past $5 \mathrm{Ma}$ normal polarity data, but it may partly be ascribed to the plate motion.

\subsection{Analysis combining with the previous data}

It is of some value to integrate the previous data (Chauvin et al., 1990; Duncan, 1975; Roperch and Duncan, 1990) into our data for further discussions. If we analyze all the available data set together after the correction for the absolute plate motion, the mean VGP locates at $88.7^{\circ} \mathrm{N}, 59.1^{\circ} \mathrm{W}$, with $\mathrm{A}_{95}=1.7^{\circ}(N=306)$. Its position is almost at the geographic pole, and the VGPs distribution is circular around the mean $\left(\alpha_{32} / \alpha_{31}=1.09\right)$. On the other hand, the distribution of the field directions does not seem to be Fisherian $\left(\alpha_{32} / \alpha_{31}=1.48\right)$. These are similar to those from our data set alone.

As for the ASD, it is calculated to be $16.2^{\circ}\left(+1.0^{\circ},-0.9^{\circ}\right)$ for the combined data set $(N=306)$, which is $1.6^{\circ}$ larger than our ASD in Table 4. It is mainly due to the number of relatively lower latitudinal VGPs reported by Roperch and Duncan (1990). If their data are excluded from the analysis, the ASD is changed to be $14.5^{\circ}\left(+0.9^{\circ},-0.9^{\circ}, N=258\right)$. This is consistent with that from our data set alone and also with the Model G for the past 5 Ma (McFadden et al., 1988, 1991). Since Roperch and Duncan (1990) mainly captured the excursional geomagnetic field, the latter ASD is more suitable for the past $5 \mathrm{Ma}$ PSV.

The combined data set $(N=258)$, excluding Roperch and Duncan (1990), also gave a time-averaged inclination of $-32.1^{\circ}\left(\alpha_{95}=2.0^{\circ}\right)$. As the corresponding GAD inclination is estimated to be $-33.6^{\circ}$, no significant inclination anomaly is observed. This situation is unchangeable, even if the plate motion is not corrected; the data set $(N=259)$ yields the TAF and GAD inclination of $-32.1^{\circ}\left(\alpha_{95}=2.0^{\circ}\right)$ and $-31.7^{\circ}$, respectively. This is probably because the platemotion correction is less important for the young-aged data $(N=82)$ from Chauvin et al. (1990). Thus we test the combined data of Duncan (1975) and our study. The resultant $\Delta I$ is calculated to be $-2.5^{\circ}\left(\alpha_{95}=2.2^{\circ}\right)$ without the plate-motion correction, whereas it is calculated to be $-0.9^{\circ}$ $\left(\alpha_{95}=2.1^{\circ}\right)$ with correction. Therefore, it is again suggested that if we treat the paleomegnetic data spanning $5 \mathrm{Ma}$, the plate motion is not negligible. It is also noted that Duncan's (1975) data does not seem to be heavily contaminated by secondary components.

\section{Conclusions}

A paleomagnetic study has been performed on $0-5 \mathrm{Ma}$ volcanic rocks from the Society Islands, French Polynesia. After plate-motion correction, 82 normal, 48 reversed, and 10 intermediate directions were observed. The combined data set $(N=130)$ gave a mean VGP at $\left(87.5^{\circ} \mathrm{N}, 4.0^{\circ} \mathrm{W}\right)$ with $\mathrm{A}_{95}=2.3^{\circ}$, and yielded an ASD around a mean of $14.6^{\circ}\left(+1.3^{\circ},-1.2^{\circ}\right)$. Although this data set does not show a significant inclination anomaly for the TAF, an apparent anomaly of $-3.4^{\circ}$ would be observed at a $95 \%$ confidence 
level $\left(\alpha_{95}=2.6^{\circ}\right)$ if the plate motion is not taken into account. This suggests that correction of the absolute plate motion is required for the precise analysis of paleodirections, especially in the lower latitude region of the Pacific Ocean. These features are still preserved, even if the previous studies are incorporated into the present analysis.

Acknowledgments. We appreciate the constructive comments of the two anonymous reviewers. This study was supported, from the 'Super Plume' project, by the Science and Technology agency of Japan.

\section{References}

Blais, S., G. Guille, R. C. Maury, H. Guillou, D. Miau, and J. Cotten, Geology and petrology of Raiatea Island (Society Islands, French Polynesia), C. R. Acad. Sci. Paris, série IIa, 324, 435-442, 1997.

Blais, S., G. Guille, H. Guillou, C. Chauvel, R. C. Maury, and M. Caroff, Geology, geochemistry and geochronology of Borabora island (Society Islands, French Polynesia), C. R. Acad. Sci. Paris, Earth Planet. Sci., 331, 579-585, 2000.

Cande, S. C. and D. V. Kent, Revised calibration of the geomagnetic polarity timescale for the Late Cretaceous and Cenozoic, J. Geophys. Res., 100 6093-6095, 1995.

Chauvin, A., P. Roperch, and R. A. Duncan, Records of geomagnetic reversals from volcanic islands of French Polynesia 2. Paleomagnetic study of flow sequence (1.2-0.6Ma) from the Island of Tahiti and discussion of reversal models, J. Geophys. Res., 95, 2727-2752, 1990.

Cox, A., Latitude dependence of the angular dispersion of the geomagnetic field, Geophys. J. R. Astr. Soc., 20, 253-269, 1970.

Duncan, R. A., Paleosecular variation at the Society Islands, French Polynesia, Geophys. J. R. Astr. Soc., 41, 245-254, 1975.

Duncan, R. A. and I. McDougall, Linear volcanism in French Polynesia, J. Volcanol. Geotherm. Res., 1, 197-227, 1976.

Gripp, A. E. and R. G. Gordon, Current plate velocities relative to the hotspots incorporating the NUVEL-1 global plate motion model, Geophys. Res. Lett., 17, 1109-1112, 1990.

Gubbins, D. and P. Kelly, Persistent patterns in the geomagnetic field over the last 2.5 Myr, Nature, 365, 829-832, 1993.

Guillou, H., S. Blais, G. Guille, R. C. Maury, A. Le Dez, and J. Cotten, Age (K-Ar) and duration of the subaerial build-up of Moorea, Raiatea and Maupiti (Society Islands, French Polynesia), Géologie de la France, 3, 29-36, 1998.

Johnson, C. and C. G. Constable, The time averaged field as recorded by lava flows over the past 5 Myr, Geophys. J. Int., 122, 489-519, 1995.

Johnson, C. and C. G. Constable, Paleosecular variation recorded by lava flows over the past five million years, Phil. Trans. R. Soc. Lond. A, 354, 89-141, 1996.
Johnson, C. and C. G. Constable, The time averaged geomagnetic field: global and regional biases for 0-5 Ma, Geophys. J. Int., 131, 643-666, 1997.

Kelly, P. and D. Gubbins, The geomagnetic field over the past 5 million years, Geophys. J. Int., 128, 315-330, 1997.

Kirschvink, J. L., The least-squares line and plane and the analysis of paleomagnetic data, Geophys. J. R. Astr. Soc., 62, 699-718, 1980.

Kono, M., Y. Hamano, T. Nishitani, and T. Tosha, A new spinner magnetometer: principles and techniques, Geophys. J. R. Astr. Soc., 67, 217$227,1984$.

Kono, M., H. Kitagawa, and H. Tanaka, Use of automatic spinner magnetometer-AF demagnetizer system for magnetostratigraphy and paleosecular variation studies (abstract), 8th Scientific Assembly IAGA, Uppsala, 1997.

Kono, M., H. Tanaka, and H. Tsunakawa, Spherical harmonic analysis of paleomagnetic data: The case of linear mapping, J. Geophys. Res., 105, 5817-5833, 2000.

Laj, C., A. Mazaud, R. Weeks, M. Fuller, and E. Herrero-Bervera, Geomagnetic reversal paths, Nature, 351, 447, 1991.

Love, J. J., Paleomagnetic volcanic data and geometric regularity of reversals and excursions, J. Geophys. Res., 103, 12435-12452, 1998.

McElhinny, M. W., P. L. McFadden, and R. T. Merrill, The time-averaged paleomagnetic field 0-5 Ma, J. Geophys. Res., 101, 25007-25027, 1996a.

McElhinny, M. W., P. L. McFadden, and R. T. Merrill, The myth of the Pacific dipole window, Earth Planet. Sci. Lett., 143, 13-22, 1996 b.

McFadden, P. L., R. T. Merrill, and M. W. McElhinny, Dipole/quadrupole family modeling of paleosecular variation, J. Geophys. Res., 93, 1158311588, 1988.

McFadden, P. L., R. T. Merrill, M. W. McElhinny, and S. Lee, Reversals of the Earth's magnetic field and temporal variations of the dynamo families, J. Geophys. Res., 96, 3923-3933, 1991.

Roperch, P. and R. A. Duncan, Records of geomagnetic reversals from volcanic islands of French Polynesia 1. Paleomagnetic study of a polarity transition in a lava sequence from the island of Huahine, J. Geophys. Res., 95, 2713-2726, 1990.

Shibuya, H., J. Cassidy, I. E. M. Smith, and T. Itaya, Paleomagnetism of young New Zealand basalts and longitudinal distribution of paleosecular variation, J. Geomag. Geoelectr., 47, 1011-1022, 1995.

Singer, B. S., K. A. Hoffman, A. Chauvin, R. S. Coe, and M. S. Pringle, Dating transitionally magnetized lavas of the late Matuyama Chron: Toward a new ${ }^{40} \mathrm{Ar} /{ }^{39} \mathrm{Ar}$ timescale of reversals and events, J. Geophys. Res., 104, 679-693, 1999.

Tanaka, H., Circular asymmetry of the paleomagnetic directions observed at low latitude volcanic sites, Earth Planets Space, 51, 1279-1286, 2000.

Y. Yamamoto (e-mail: yuhji-yamamoto@aist.go.jp), K. Shimura, H. Tsunakawa, T. Kogiso, K. Uto, H. G. Barsczus, H. Oda, T. Yamazaki, and E. Kikawa 